\title{
Immunohistochemical profile of neurotrophins in human cranial dura mater and meningiomas
}

\author{
MARCO ARTICO $^{1}$, ELENA BRONZETTI ${ }^{2}$, ELENA POMPILI ${ }^{2}$, BRUNELLA IONTA ${ }^{1}$, \\ VALENTINA ALICINO ${ }^{1}$, ANNA D'AMBROSIO ${ }^{2}$, ANTONIO SANTORO ${ }^{3}$, \\ FRANCESCO S. PASTORE ${ }^{4}$, ILIA ELENKOV ${ }^{2}$ and LORENZO FUMAGALLI ${ }^{2}$ \\ ${ }^{1}$ Department of Otorhinolaringology, Audiology and Phoniatry 'G. Ferreri'; \\ ${ }^{2}$ Department of Cardiovascular and Respiratory Sciences, Section of Experimental Morphology; \\ ${ }^{3}$ Department of Neurological Sciences, Neurosurgery, University of Rome 'La Sapienza'; \\ ${ }^{4}$ Division of Neurosurgery, University of Rome 'Tor Vergata', Rome, Italy
}

Received November 21, 2008; Accepted February 12, 2009

DOI: 10.3892/or_00000363

\begin{abstract}
The immunohistochemical profile of neurotrophins and their receptors in the human cranial dura mater was studied by examining certain dural zones in specimens harvested from different regions (frontal, temporal, parietal and occipital). Dural specimens were obtained during neurosurgical operations performed in ten patients for surgical treatment of intracranial lesions (meningiomas, traumas, gliomas, vascular malformations). The dural fragments were taken from the area of the craniotomy at least $8 \mathrm{~cm}$ from the lesion as well as from the area in which the meningioma had its dural attachment. Immunohistochemical characterization and distribution of neurotrophins, with their receptors, were analyzed. The concrete role played by these neurotrophic factors in general regulation, vascular permeability, algic responsivity and release of locally active substances in the human dura mater is still controversial. Our study revealed a general structural alteration of dural tissue due to the invasivity of meningiomatous lesions, together with an improved expression of brain derived neurotrophic factor (BDNF) in highly proliferating neoplastic cells and an evident production of nerve growth factor (NGF) in inflammatory cells, suggesting that BDNF has a role in supporting the proliferation rate of neoplastic cells, while NGF is involved in the activation of a chronic inflammatory response in neoplastic areas.
\end{abstract}

Correspondence to: Professor Marco Artico, Deptartment of Otorhinolaringology, Audiology and Phoniatry 'G. Ferreri', University of Rome 'La Sapienza', V. le del Policlinico 155, 00161 Rome, Italy

E-mail: marco.artico@uniroma1.it

Key words: neurotrophins, human, dura mater, immunohistochemistry, meningioma

\section{Introduction}

Meningiomas are the most common benign intracranial tumors, but some meningiomas show malignancy with invasion into the surrounding structures, as well as a high recurrence rate and extracranial metastases (1). Meningiomas arise from arachnoidal cells, most of which lie in close proximity to the venous sinuses: in fact, this is the most common site for meningioma formation. They are most frequently attached to the dura mater over the superior parasagittal surface of the frontal and parietal lobes, along the sphenoid ridge, in the olfactory grooves, the Sylvian region, superior cerebellum along the falx cerebri, cerebellopontine angle and spinal cord. The tumor is usually well-circumscribed, with the base lying on the dura mater.

Histologically, the cells are relatively uniform, with a tendency to form highly-circumscribed whorls and to generally disrupt the distribution and the structure of collagen fibers (main component of dural tissue) and to originate 'psammoma bodies' (laminated concretions) which can calcify and are often strongly vascularized.

The remarkable proliferation rate and vascularisation which determine a rapid replacement of normal dural tissue by the neoplastic cells, is regulated by several different growth factors, such as NGF related to an increased activation of the inflammatory cells at the beginning of the neoplastic invasion and BDNF, involved in the increased activation of the tumoral cells.

Neurotrophins (NTs), also known as neurotrophic factors, constitute a family of dimeric proteins working as polypeptidic growth factors and acting like extracellular ligands. NTs, including NGF, BDNF, NT-3, NT-4, are involved in vertebrate neuronal cell development, differentiation, survival and functional activities.

Neurotrophins are also involved in the modulation of adult central nervous system functions and organization, as well as in the neural control of different activities related to vegetative innervation of several organs (2-6). A definite role of NTs in the dural and leptomeningeal compartments is not well 
ascertained but a previous study on the expression of mRNAs for NTs revealed high levels of NT3 and NGF in the normal rat dura mater (7). Our observations on the localization and possible roles in different non-neuronal tissues such as lymphoid, lung and prostate (8-11) encouraged us to persist in our experimental investigations in order to find a possible involvement of NTs in dura mater, both in physiological and pathological conditions (meningiomas).

Our study investigated the immunohistochemical localization of NTs and their receptors both in normal human cranial dura mater and in the dural tissue close to the dural attachment of the meningiomatous mass, focusing on a possible correlation between the dural distribution of these neurotrophic factors and the physiopathological mechanisms involved in meningioma development.

\section{Materials and methods}

Patients. Neurosurgical operations were performed on ten adult patients (age range 30-75 years) and 2 fragments (for each subject) of dura mater were obtained from occipital (1 basal -posterior cranial fossa and 1 apical), frontal ( 1 basal -anterior cranial fossa- and 1 apical -parasagittal-), parietal (1 parasagittal and 1 convexity), and temporal (1 basal -middle cranial fossa- and 1 apical- corresponding with the dural area located at least $1 \mathrm{~cm}$ behind the temporal crest) regions.

The dural fragments were taken from the craniotomy area at least $8 \mathrm{~cm}$ distant from the lesion or in the areas close to the meningioma (four meningotheliomatous-psammomatous and five transitional). Human dural tissue was surgically removed and processed for immunohistochemical and RTPCR analysis. Experiments were performed in compliance with the Italian laws and guidelines concerning the informed consent of the patients. The following molecules were investigated: nerve growth factor (NGF), brain-derived neurotrophic factor (BDNF), NT-3, NT-4 and the neurotrophin receptors such as tyrosine kinase A (TrKA), tyrosine kinase B (TrKB), tyrosine kinase C (TrKC) and protein 75 (p75).

Immunohistochemistry. Small fragments of dural tissue were washed in PBS, fixed in $10 \%$ formalin and embedded in paraffin according to a standard procedure. Serial $10-\mu \mathrm{m}$ thick sections were cut using a microtome, mounted on gelatincoated slides and processed for immunohistochemistry. To study the immunolocalization of neurotrophins and their own receptors, the antibodies used were: i) rabbit anti-nerve growth factor (anti-NGF) polyclonal antibody (Santa Cruz, CA, USA). It displayed $<1 \%$ cross-reactivity against recombinant human NT-3, NT-4 and BDNF; ii) rabbit antityrosine kinase A (anti-TrKA) polyclonal antibody (Santa Cruz). It recognized an epitope corresponding to amino acids 763 to 777 , mapping adjacent to the carboxy-terminus of human TrKA p140; iii) goat polyclonal antibody to human p75 NT receptor (Santa Cruz). It recognized the amino acid sequence mapping the carboxy-terminus of the p75 NT receptor precursor of human origin; iv) rabbit anti-brain derived neurotrophic factor (anti-BDNF) polyclonal antibody (Santa Cruz). It recognized the amino-terminal of mouse BDNF; v) rabbit anti-tyrosine kinase B (anti-TrKB) polyclonal antibody (Santa Cruz). It recognized an epitope corresponding to amino acids 794 to 808 of mouse TrKB p145; vi) rabbit anti-neurotrophin 3 (anti-NT-3) polyclonal antibody (Santa Cruz). It was raised against the amino-terminal of mouse NT3; vii) rabbit polyclonal anti-tyrosine kinase $\mathrm{C}$ (anti-TrKC) antibody (Santa Cruz). It recognized an epitope corresponding to amino acids 798 to 812 of porcine TrKC p140. The immunohistochemical recognition of macrophages was performed using a mouse monoclonal anti-human CD68 antibody (Dako Cytomation, Denmark). Incubation with primary antibodies was performed overnight at $4^{\circ} \mathrm{C}$ at a final concentration of $2-5 \mu \mathrm{g} / \mathrm{ml}$. Optimal antisera dilutions and incubation times were assessed in a series of preliminary experiments. After exposure to the primary antibodies, slides were rinsed twice in phosphate buffer and incubated (90 min at room temperature) with the appropriate secondary antibody conjugated to horseradish peroxidase (HRP) (final dilution 1:100). The secondary antibody-HRP linked against rabbit immuno-globulins was purchased from Boehringer Mannheim GmbH (Mannheim, Germany), while secondary antibodies-HRP linked against mouse and goat immunoglobulins were from Sigma (Sigma Chemicals Co, St. Louis, MO, USA). After a further wash with phosphate buffer, slides were treated with $0.05 \%$ 3,3-diaminobenzidine and $0.1 \%$ $\mathrm{H}_{2} \mathrm{O}_{2}$. Finally, sections were counterstained with Mayer's hematoxylin and observed by using a light microscope. To block endogenous peroxidase activity, slides were pretreated with $3 \% \mathrm{H}_{2} \mathrm{O}_{2}$, whereas the non-specific binding of immunoglobulins was prevented by adding $3 \%$ fetal calf serum to the incubation medium. Negative control experiments were done: i) by omitting the primary antibody; ii) by substituting the primary antibody with equivalent amount of non-specific immunoglobulins; iii) by pre-incubating the primary antibody with the specific blocking peptide (antigen/antibody $=5$ according to supplier's instructions). In preliminary experiments, immunohistochemistry was also performed on frozen sections of human dural tissue. No differences were found in the intensity or distribution of immunostaining using the two types of sections, but microanatomical details were better preserved in paraffin-embedded material. The intensity of immune reaction was assessed microdensitometrically by an IAS 2000 image analyzer (Delta Sistemi, Rome, Italy) connected via a TV camera to the microscope. The system was calibrated taking the background obtained in sections exposed to non-immune serum as zero. Ten $100 \mu \mathrm{m}^{2}$ areas were delineated in each section by a measuring the diaphragm. Quantitative data of the intensity of the immune staining were analyzed statistically by analysis of variance (ANOVA) followed by Duncan's multiple range test as a post hoc test. The parameters examined by quantitative analysis were: i) the dural regions from which the fragments were taken $(\mathrm{A}=$ basal region of the cranial dura, $\mathrm{B}=$ apical region of the cranial dura); ii) examined zones (I = vasal zone, strictly corresponding to vasal perimeter; II = perivasal zone, corresponding to a ring $100 \mu \mathrm{m}$ in diameter around the vessel; $\mathrm{III}=$ dural or intervasal zone, at least $50 \mathrm{~mm}$ away from the nearest vessel boundary); iii) distribution of the immunoreactivity. These values were transformed into a single number expressed as conventional unit, including the standard deviation. This number can be read on the display of the Quantimet 500 image analyzer. 


\section{normal dura mater dura/meningioma}

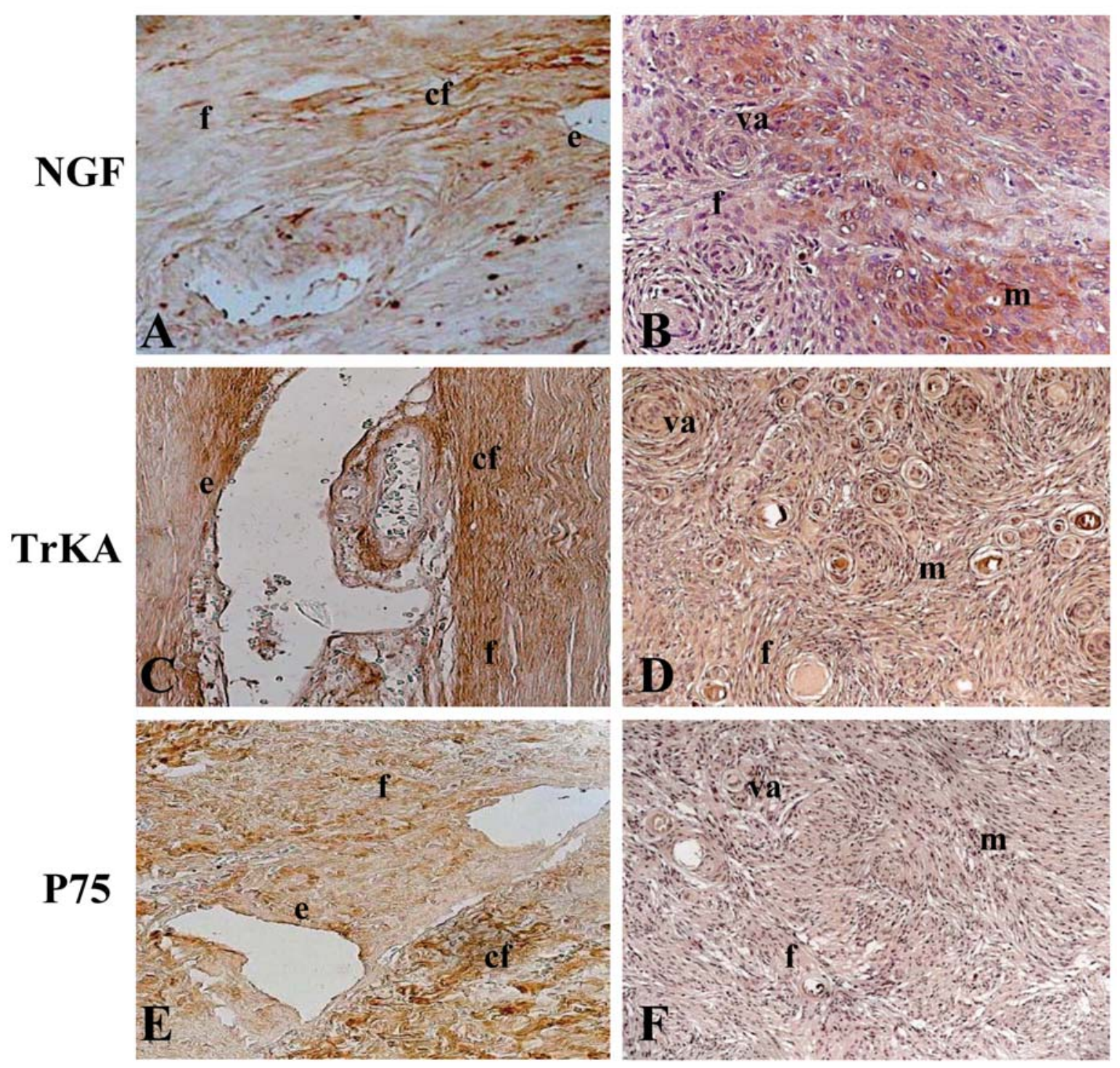

Figure 1. Micrographs of NGF immunostaining in normal human dura mater (A) and in the transitional meningioma (B)/surrounding dural tissue, $\mathrm{x} 40$. The immunoreactivity for NGF is evident in close proximity to the blood vessel endothelium (e), in fibroblasts (f) and collagen fibers (cf) in the normal dura mater and in dural tissue close to meningioma. The immunostaining for NGF in the transitional meningioma (B) is more evident in the aggregated macrophages (m) and fibroblasts (f). Micrographs of TrKA immunostaining normal human dura mater (C) and in the meningotheliomatous-psammomatous meningioma (D)/surrounding dural tissue. In micrographs (C) a vessel with its endothelium (e) is clearly visible. Moreover, another small vessel within the wall of the vessel (vasum vasorum) may be observed and an intense immunoreactivity for TrkA is present in the wall of this vessel and in the aggregated fibroblasts (f) and collagen fibers (cf). Micrographs of TrKA reactivity in a meningotheliomatous-psammomatous meningioma (D) and surrounding dural tissue, macrophages and the endothelium of the vessels are characterized by a moderate immunoreaction. Micrographs of the immunoreactivity for p75NTR in the normal dura (E) and in a transitional meningioma (F)/surrounding dural tissue. P75NTR shows a moderate immunoreaction in the endothelium, a weak expression in macrophages ( $\mathrm{m})$, fibroblasts (f) and collagen fibers (cf).

$R T-P C R$. Total RNA was isolated from human dural tissue by using TRIzol reagent (Invitrogen, Carlsbad, CA, USA) according to the manufacturer's instructions. cDNA was synthezised from $1 \mu \mathrm{g}$ total RNA in a final reaction volume of $20 \mu \mathrm{l}$. Briefly, a mixture of total RNA, oligo (dT), dNTP mix and DEPC-treated distilled water was preincubated for $5 \mathrm{~min}$ at $65^{\circ} \mathrm{C}$; then SuperScript III reverse transcriptase (200 U), RNase Ribonuclease Inhibitor, DTT and buffer (250 $\mathrm{mM}$ Tris $\mathrm{pH} 8.3,375 \mathrm{mM} \mathrm{KCl}, 15 \mathrm{mM} \mathrm{MgCl}_{2}$ ) were added to the mixture and incubation was continued for $45 \mathrm{~min}$ at $50^{\circ} \mathrm{C}$. Superscript III was disactivated by heating for $15 \mathrm{~min}$ at $70^{\circ} \mathrm{C}$. All reagents were from Invitrogen. The resulting cDNA (3-5 $\mu \mathrm{l}$ ) was amplified by polymerase chain reaction (PCR). Each PCR tube contained the following reagents: $0.2 \mathrm{mM}$ of both sense and antisense primers, 3-5 ml template cDNA, $0.2 \mathrm{mM}$ 4-dNTP mix (Invitrogen), $2.5 \mathrm{U}$ Platinum Taq DNA polymerase (Invitrogen) and $1 \mathrm{X}$ reaction buffer (Invitrogen). $\mathrm{MgCl}_{2}$ was added at a final concentration of $1 \mathrm{mM}$ for BDNF, NT3, TrKA and TrKB and at a final concentration of $1.5 \mathrm{mM}$ for NGF, TrKC and $\mathrm{p} 75$. The final volume was $50 \mathrm{ml}$. The PCR primers used for amplifying neurotrophins and their receptors (M-Medical, 


\section{normal dura mater dura/meningioma}

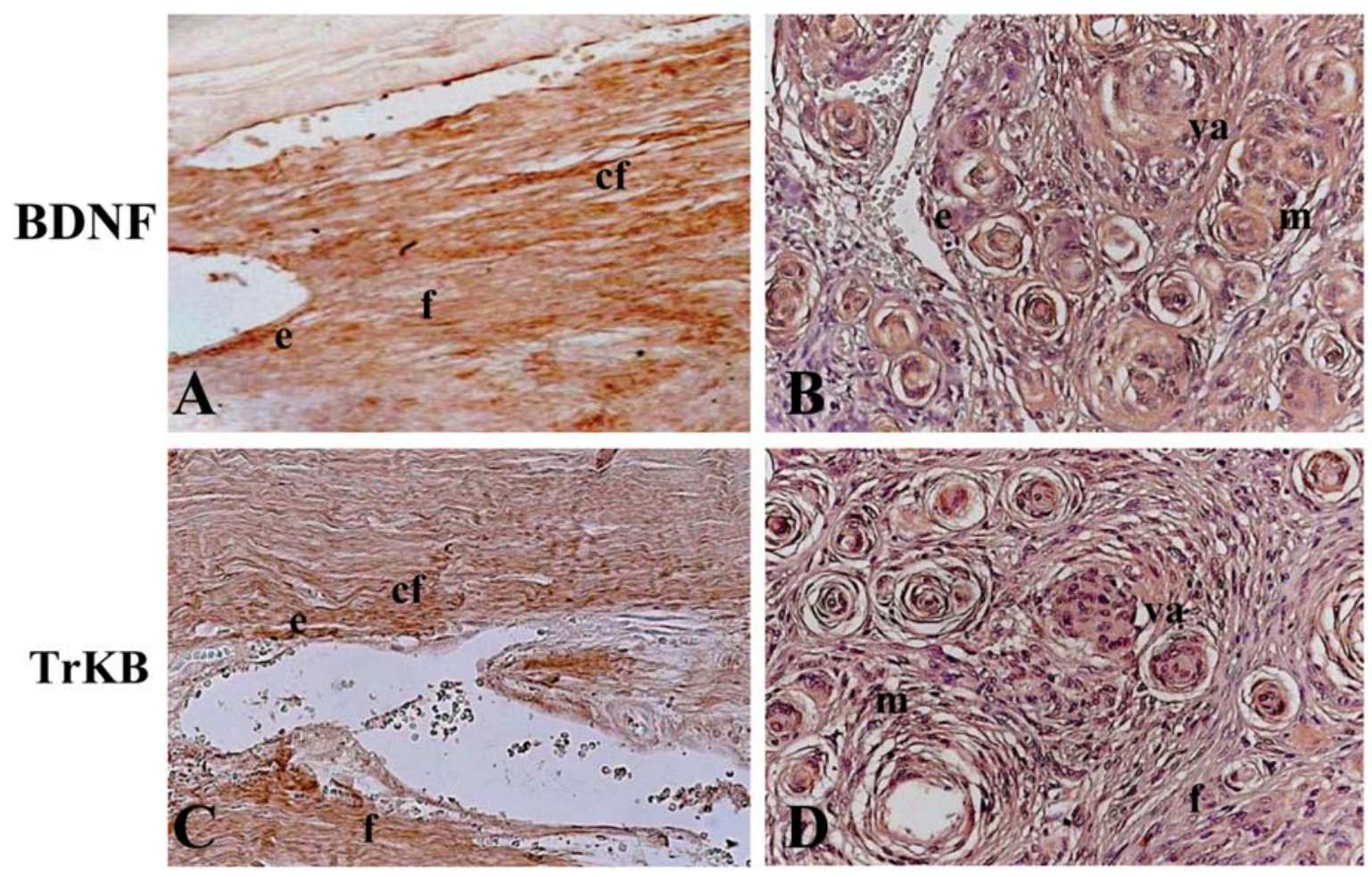

Figure 2. Micrographs of BDNF immunoreactivity in normal human dura mater (A) and in meningotheliomatous-psammomatous meningioma (B)/surrounding dural tissue, $\mathrm{x} 40$. An appreciable immunoreactivity is visible in fibroblasts (f) and collagen fibers (cf), as well as in the endothelium of vessels (e). An analogous positive immunoreactivity for BDNF is markedly visible in the vorticoid areas (va) and in the endothelium (e) and moderate immunoreactivity may be observed in the aggregates of macrophages $(\mathrm{m})$. The TrkB immunoreactivity appears to be moderate or weak in the fibroblasts (f) and collagen fibers (cf) of the dura mater $(\mathrm{C})$, but is more intense in the vorticoid areas (va) of the transitional meningioma (D).

Florence, Italy) were: for NGF forward TCATCATCCCAT CCCATCTT, reverse CTTGACAAAGGTGTGAGTCG; for BDNF forward AGCCTCCTCTGCTCTTTCTGCTGGA, reverse CTTTTGTCTATGCCCCTGCAGCCTT; for NT3 forward TTTCTCGCTTATCTCCGTGGCATCC, reverse GGCAGGGTGCTCTGGTAATTTTCCT; for NT4 forward GCTGTGGACTTGCGTGG, reverse GCCCGCACATAGG ACTG; for TrKA forward TCTTCACTGAGTTCCTGGAG, reverse TTCTCCACCGGGTCTCCAGA; for TrKB forward AAGACCCTGAAGGATGCCAG, reverse AGTAGTCAGT GCTGTACACG; for TrKC forward GGAAAGGTCTTCCT GGCCGAGTGC, reverse GCTTTCCATAGGTGAAGATC TCCC; for p75 forward TGGACAGCGTGACGTTCTCC, reverse GATCTCCTCGCACTCGGCGT. The specificity of the primers was verified by searching for every possible homology to cDNAs of unrelated known proteins in the NCBI database. PCR reaction consisted of incubation for $2 \mathrm{~min}$ at $94^{\circ} \mathrm{C}$ followed by $30-35$ cycles of incubation at $94^{\circ} \mathrm{C}$ for $30 \mathrm{sec}, 56^{\circ} \mathrm{C}$ (for NGF, NT4, TrKA and TrKB) or $62^{\circ} \mathrm{C}$ (for BDNF, NT3, p75 and TrKC) for $30 \mathrm{sec}$ and $72^{\circ} \mathrm{C}$ for 1 min. PCR products were separated by agarose gel electrophoresis (Submarine Agarose Gel Unit, Hoefer, San Francisco, CA, USA) and visualized using a digital gel documentation system (GelDoc 2000 System/Quantity One Software; Bio-Rad Laboratories, Hercules, CA, USA).

\section{Results}

Immunohistochemistry. Immunoreactivity for neurotrophins and their receptors has been observed both in the normal dura mater and in the dural tissue close to the meningiomatous area. The sections harvested from normal dura mater generally revealed a marked immunoreactivity in the endothelium of the dural vessels, in collagene fibers and in fibroblasts.

Immunoreaction for NGF was clearly demonstrated in fibroblasts and collagen fibers and also appeared to the same degree in the endothelium of the dural vessels (Fig. 1A). In the same way TrkA immunoreactivity was strongly marked in the dural vessel endothelium, in fibroblasts and collagen fibers (Fig. 1C). A moderate immunoreactivity for the p75NTR was demonstrated in the dural vessel endothelium and weakly expressed in fibroblasts and collagene fibers (Fig. 1E). BDNF immunoreactivity was appreciable in the dural vessel wall, in fibroblasts and collagen fibers (Fig. 2A) and in the same compartments we observed a reaction for TrkB (Fig. 2C). Moderate immunoreactivity was demonstrated for NT-3 and NT-4 in the previous compartments (Fig. 3A and C). On the other hand, TrkC immunoreactivity was appreciable in the same areas (Fig. 3E ).

Meningiomatous sections revealed a general structural alteration of collagen fiber with compressed fibroblasts within 


\section{normal dura mater dura/meningioma}

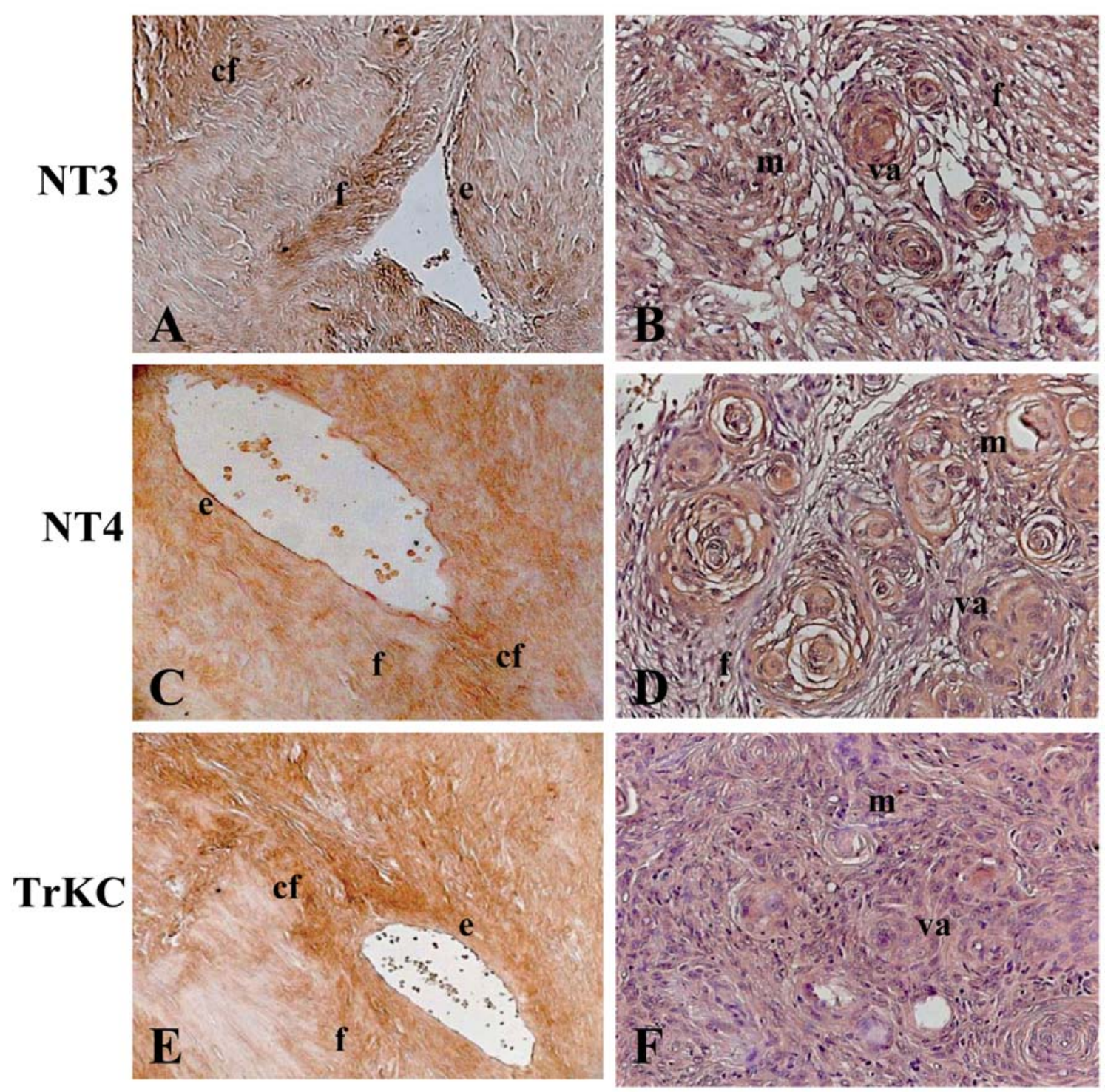

Figure 3. Micrographs of NT-3 and NT-4 immunoreactivity in normal dura mater (A and C), in transitional meningioma (B) and in meningotheliomatouspsammomatous meningioma (D)/surrounding dural tissue, x40. The immunoreactivity for NT-3 appears weak or moderate in all the observed structures both in the normal dura mater (A) and in the transitional meningioma (B)/surrounding dural tissue. A moderate immunoreactivity for NT-4 may be observed in the normal dura mater $(\mathrm{C})$ while the immunoreactivity appears to be appreciable in the meningiomatous vorticoid areas (va). Micrographs of the immunoreaction for TrkC in the normal dura mater (E) and in the meningotheliomatous-psammomatous meningioma (F)/surrounding dural tissue; the immunoreactivity appears to be moderate both in fibroblasts (f) and collagen fibers (cf) and in the endothelium of the vessels (e) in the normal dura mater. An appreciable immunoreactivity is visible in the vorticoid areas (va) of the meningioma (F).

the well-circumscribed whorls, which completely replaced the normal dural tissue. We also observed an evident immunoreaction in the dural vessel wall, in the aggregates of fibroblasts/macrophages and in the whorls (vorticoid areas), which may calcify in some histotypes, thus forming laminar concentrically arranged masses named psammomatous bodies. The macrophage aggregates showed a strong immunoreactivity for NGF and TrkA, while a moderate reaction was demonstrated for blood vessel endothelium (Fig. 1B and D); P75NTR immunoreactivity was, on the contrary, very weak in the same compartments (Fig. 1F). The neoplastic cells in the whorls showed a remarkable immunoreactivity for BDNF and TrkB (Fig. 2B and D), thus confirming our previous results in other neoplastic tissues, such as lung and prostate $(11,12)$, in which we demonstrated a significant role of BDNF in the increased proliferation rate of neoplastic lesions.

NT-3 was weak and NT-4 immunoreaction was moderate in the neoplastic cells of the whorls (Fig. 3B and D), in the dural vessel wall and in the macrophage aggregates. Similar result was observed for TrkC and p75 (Fig. 3F).

$R T-P C R$. RT-PCR analysis confirmed the findings of the immunohistochemical investigation both in the normal dura and in the dural area close to meningiomatous tissue. We observed a strong expression of specific transcripts for BDNF and TrKB, moderate for NT-4 in the meningiomatous 


\section{RT-PCR in normal dura mater and meningioma specimens}

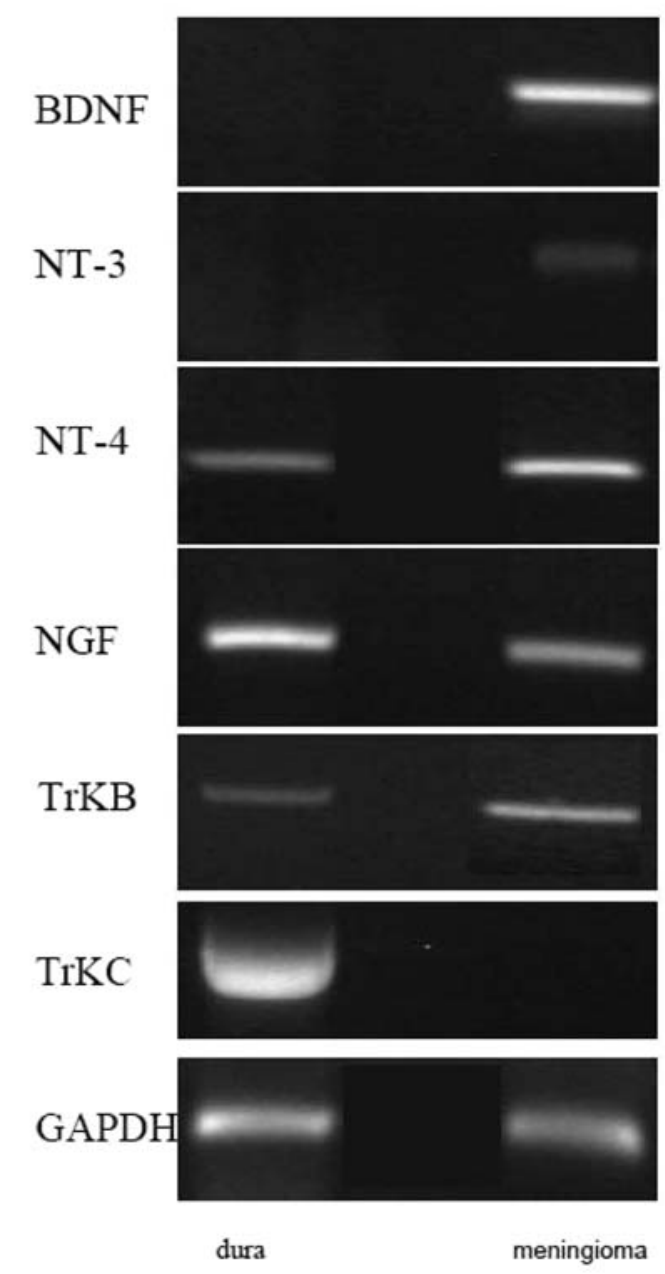

Figure 4. RT-PCR comparative analysis between normal dura mater and meningioma/surrounding dural tissue. Specific transcripts for NGF, BDNF, NT-3, NT-4 and high affinity receptors TrkB and TrkC.

tissue and a relevant expression of NGF in the dura mater close to the tumor lesion (Fig. 4).

\section{Discussion}

Notwithstanding the common (mesodermal) origin of all the meninges there are, in any case, relevant differences in the biological behaviour of the leptomeninx (arachnoid and pia mater) and pachimeninx (dura mater). In fact, only the leptomeninx contains the meningoblasts (cells responsible for the genesis and the development of meningiomas). The dura mater does not play a role in the onset of meningiomas (it only rarely develops into other unusual tumors such as some sarcomas) but often has a close relationship with the meningiomatous lesions which are characterized by a close contiguity with the pachimeninx itself, with the exception of those rare meningiomas without dural attachment that are often intraventricular. These close relationships between meningiomas and dura mater persuaded us to investigate the
Table I. Normal dura mater.

\begin{tabular}{lccc}
\hline & $\begin{array}{c}\text { Fibroblasts } \\
\text { collagen fibers }\end{array}$ & Macrophages & $\begin{array}{c}\text { Blood vessels } \\
\text { endothelium }\end{array}$ \\
\hline NGF & ++ & \pm & + \\
BDNF & + & - & + \\
NT-3 & \pm & - & \pm \\
NT-4 & \pm & - & \pm \\
TrKA & + & \pm & + \\
TrKB & \pm & - & \pm \\
TrKC & + & - & + \\
P75NTR & + & - & \pm \\
\hline
\end{tabular}

\pm , weak immunoreactivity; + , moderate immunoreactivity and ++ strong immunoreactivity.

Table II. Meningioma/surrounding dural tissue.

\begin{tabular}{lcccc}
\hline & $\begin{array}{c}\text { Fibroblasts } \\
\text { collagen } \\
\text { fibers }\end{array}$ & Macrophages & $\begin{array}{c}\text { Vorticoid } \\
\text { areas }\end{array}$ & $\begin{array}{c}\text { Blood } \\
\text { vessels } \\
\text { endothelium }\end{array}$ \\
\hline NGF & \pm & ++ & \pm & + \\
BDNF & \pm & \pm & ++ & + \\
NT-3 & - & \pm & $+/+$ & \pm \\
NT-4 & - & \pm & $+/+$ & \pm \\
TrKA & \pm & + & \pm & \pm \\
TrKB & - & \pm & ++ & \pm \\
TrKC & - & \pm & + & \pm \\
P75NTR & - & - & \pm & \pm \\
\hline
\end{tabular}

\pm , weak immunoreactivity; + , moderate immunoreactivity and ++ strong immunoreactivity.

immunohistochemical profile of neurotrophins in the normal dura and in the dura adjacent to the meningiomatous lesion in order identify any differences in the localization of neurotrophins in that compartments and to demonstrate a possible direct role in tumorigenesis and in the progression of these specific neoplastic lesions.

Numerous studies have stressed the relevance of the morphological structure of dural innervation, in fact some interesting investigations in rats (12-17) have focused on the peptidergic, catecholaminergic and nitroxidergic innervation of dura mater encephali (18), revealing a possible relationship with the etiopathogenesis of headache and dural pain $(13,15,19,20)$.

The dura mater shows a high density of sympathetic nerve fibers and an impressive population of mast cells, mainly perivascular. It also receives significant sensory projections from the trigeminal system. The presence of these three elements in the meningeal layer suggests a relevant functional interaction between the nervous and the immune system, both mediated by neurotrophic factors $(6,13,14,20,21)$. 
It is well known that NGF has an inflammatory role and its increase is directly related to inflammation and diseases of the immune system $(4,6,22)$, probably due to the direct action of NGF on mast cells and sensory neurones, as proposed by Woolf and Ma (19). Interestingly, dura mater cells contain high levels of NGF and TrKC, and moderate levels of BDNF and NT-4, suggesting that NGF is essential in preserving the integrity of the mast cell/nerve unit (7).

Neurotrophins (NTs) are neurotrophic signalling polypeptides which include nerve growth factor (NGF), brain derived growth factor (BDNF), neurotrophin-3 (NT-3), NT-4/5 and NT-6, the latter apparently being specific to fish $(2,3)$. The biological effects of NTs are mediated by the binding with two families of membrane receptors, the high affinity tyrosine kinase (TrK) and low affinity $\mathrm{p} 75$ receptor (p75NT receptor) (2). The TrK family includes TrKA, TrKB and TrKC receptors, whereas p75NT receptor belongs to the trans-membrane molecules serving as receptor for tumor necrosis factor and cytokines (3). TrKA is specifically activated by NGF, whereas TrKB and TrKC are primarily receptors for BDNF and NT-3 respectively (3).

The physiological role of NTs in the development, maintenance and regeneration of the sympathetic and sensory nervous system has been well established (23-25) and mainly NGF induces differentiation and decreases growth rate in a variety of neoplastic cells from neurogenic and non-neurogenic origin (26-29). There is increasing evidence that NTs may act together with a paracrine mechanism in the regulation of the functional activity of neuronal and non-neuronal structures (30).

We have investigated the possible role of NTs in the physiopathology of dura diseases, especially in neoplastic lesions.

NGF showed an appreciable expression rate in the normal dura mater and in the dura close to the meningiomatous tissue, but the distribution of this growth factor and its high affinity receptor TrkA in the whorls (vorticoid areas) appeared markedly different. In fact NGF and TrKA resulted markedly expressed in macrophages, thus confirming their functional involvement in the persistence of a chronic inflammatory condition in the tumoral lesion. BDNF and TrKB were, on the contrary, strongly expressed in the vorticoid areas and this finding suggests that they play a direct role in the development and progression of the meningiomatous cells.

NGF is found in many tissues and cells including the brain, meninges, and cerebrospinal fluid (32), while BDNF is expressed throughout the central nervous system as well as in the periphery, mainly in sensory neurons expressing NGF receptors. Peripheral noxious stimuli, such as trauma or inflammation, up-regulate NGF in the affected tissue, as well as BDNF in the sensory ganglia. However, while NGF contributes specifically to inflammation and neuropathic pain inducing the sensitization of peripheral nociceptors, BDNF released within the central nervous system acts as a pain modulator contributing to the central sensitization, probably via the activation of NMDA receptors (22).

BDNF is directly produced by meningeal cells $(22,25,28,31-33)$ together with pro-inflammatory cytokines, reducing cellular apoptosis $(1,33)$ and promoting cellular survival in an autocrine or paracrine manner in $\operatorname{TrKB}$ expressing human neuroblastoma $(30,33,34)$.

The expression of p75 appeared moderate in the normal dura mater, but weak both in the meningiomatous tissue and in the surrounding dural compartment, because this receptor is lost during tumor progression (28).

RT-PCR analysis confirmed our immunohistochemical observations: we have described a moderate expression of NGF and its high-affinity receptor TrKA in normal dura mater, in the meningioma tissues and in the surrounding dural compartments. This finding encouraged us to believe in a direct role of NGF/TrKA in the maintenance of a chronic inflammatory condition, necessary to the beginning and the consequent progression of the neoplastic lesion. On the other hand, other neurotrophins such as BDNF, its high-affinity TrKB and NT-4 were markedly expressed both in the meningioma specimens and in the surrounding dural tissue. This finding suggests a direct involvement of BDNF/NT-4 in the development and proliferation of the tumor cells (see Tables I and II).

The data described above confirmed our previous experimental findings (8-11), hence improving our knowledge on the role of the neurotrophins in the regulation of the meningeal microenvironment and their direct participation in the neoplastic transformation and cellular survival, both essential aspects of the malignant development.

\section{References}

1. Nagashima G, Aoyagi M, Yamamoto M and Yamamoto S: P53 overexpression and proliferative potential in malignant meningiomas. Acta Neurochir 141: 53-61, 1999.

2. Barbacid M: The TrK family of neurotrophin receptors. J Neuroimmunol 25: 1386-1403, 1994.

3. Kaplan DR and Miller FD: Signal transduction by the neurotrophin receptors. Curr Opin Cell Biol 9: 213-221, 1997.

4. Vega JA, Garcia-Suarez O, Hannestad J, Perez-Perez M and Germanà $\mathrm{A}$ : Neurotrophins and the immune system. (Review) $\mathrm{J}$ Anat 203: 1-19, 2003.

5. Otten U, Marz P, Heese K, Hock C, Kunz D and Rose-John S: Cytokines and neurotrophins interact in normal and diseased states. (Review) Ann NY Acad Sci 917: 322-330, 2000.

6. Stanisz AM and Stanisz JA: Nerve growth factor and neuroimmune interactions in inflammatory diseases. Ann NY Acad Sci 917: 268-272, 2000.

7. Timmusk T: Expression of mRNA for neurotrophins and their receptors in the rat choroid plexus and dura mater. Neuroreport 6: 1997-2000, 1995.

8. Bronzetti E, Artico M, Lo Vasco VR, Felici LM, Bosco S, Magliulo G, Pompili E and Fumagalli L: Expression of neurotransmitters and neurotrophins in human adenoid tissue. Int J Mol Med 15: 921-928, 2005.

9. Bronzetti E, Artico M, Pompili E, Felici LM, Stringaro A, Bosco S, Magliulo G, Colone M, Arancia G, Vitale M and Fumagalli L: Neurotrophins and meurotransmitters in human palatine tonsils: an immunohistochemical and RT-PCR analysis. Int J Mol Med 18: 49-58, 2006.

10. Ricci A, Felici LM, Mariotta S, Mannino F, Schmid G, Terzano C, Cardillo G, Amenta F and Bronzetti E: Neurotrophins and neurotrophin receptor protein expression in the human lung. Am J Respir Cell Mol Biol 30: 12-19, 2004.

11. Bronzetti E, Artico M, Forte F, Pagliarella G, Felici LM, D'Ambrosio A, Vespasiani G and Bronzetti B: A possible role of BDNF in prostate cancer detection. Oncol Rep 19: 969-974, 2008.

12. Goadsby PJ and Edvinsson L: Human in vivo evidence for trigeminovascular activation in cluster headache. Neuropeptide changes and effects of acute attacks therapies. Brain 117: 427-434, 1994. 
13. Artico M, De Santis S and Cavallotti C: Cerebral dura mater and cephalalgia: relationships between mast cells and cathecolaminergic nerve fibers in the rat. Cephalalgia 18: 183-191, 1998.

14. Keller JT, Dimlich RVW and Zuccarello M: Relationship of trigeminovascular and sympathetic nerve fibers to mast cells in the dura mater of the rat. J Cer Blood Flow Metab 9: S685, 1989.

15. Olesen J and Edvinsson L: Basic Mechanisms of Headache. Elsevier, Amsterdam, pp1492, 1988.

16. Keller JT, Marfurt CF, Dimlich RVW and Tierney BE: Sympathetic innervations of the supratentorial dura mater of the rat. J Comp Neurol 290: 310-321, 1989.

17. Fricke B, Duering von M and Andres KH: Topography and immunocytochemical characterization of nerve fibers in the leptomeningeal compartments of the rat. A light- and electronmicroscopical study. Cell Tissue Res 287: 11-22, 1997.

18. Andres KH, von Duering M, Muszynski K and Schmidt RF: Nerve fibers and their terminals of the dura mater encephali of the rat. Anat Embryol 175: 289-301, 1987.

19. Woolf CJ and Ma QP: Peripheral cell types contributing to the hyperalgesic action of nerve growth factor in inflammation. J Neurosci 16: 2716-2723, 1996.

20. Dimitriadou, et al: Functional relationships between sensory nerve fibers and mast cells of dura mater in normal and inflammatory conditions. Neuroscience 7: 829-839, 1997.

21. Lomen HC and Shooter EM: Widespread neurotrophin receptor expression in the immune system and other non-neuronal rat tissue. J Neurochem 64: 1780-1789, 1995.

22. Blandini F, Rinaldi L, Tassorelli C, et al: Peripheral levels of BDNF and NGF in primary headaches. Cephalalgia 26: 136-142, 2005.

23. Snider WD: Functions of the neurotrophins during nervous system development: what the knockouts are teaching us. Cell 77: 627-638, 1994

24. Weiner HL: The role of growth factor receptors in central nervous system development and neoplasia. Neurosurgery 35: 179-194, 1995
25. Barbara E, Lazio MD, Lawrence S and Chin M: Growth factors in central nervous system development and tumorigenesis. Neurosurg Focus 3: 1997.

26. Yaeger MJ, Koestner A and Marushige Y: The reverse transforming effects of nerve growth factor on five human neurogenic tumor cell lines: in vitro results. Acta Neuropathol 83: 72-80, 1991.

27. Jagodzinski PP, Kaczmarski W and Zukiel R: Nuclear accumulation of nerve growth factor and its receptor in meningioma and glioma tumoury cells. Bulletin of the Polish Academy of Sciences/Biological Sciences 40: 145-152, 1992.

28. Cross $M$ and Dexter TM: Growth factors in development, transformation, and tumorigenesis. Cell 64: 270-280, 1991.

29. Kruttgen A, Schneider I and Weis J: The dark side of the NGF family: Neurotrophin in neoplasias. Brain Pathol 16: 304-310, 2006.

30. Lewin GR and Renz H: Abundant production of brain-derived neurotrophic factor by adult visceral epithelia implications for paracrine and target-derived neurotrophic functions. Am J Pathol 155: 1183-1193, 1999.

31. Damien B: Differential Gene expression during meningealmeningococcal interaction: evidence for self-defense and early release of cytokines and chemokines. Infect Immun 69: 2718-2722, 2001.

32. Tsujino K: Establishment and characterization of cell lines derived from a transplantable rat malignant meningioma: morphological heterogeneity and production of nerve growth factor. Acta Neuropathol 93: 461-470, 1997.

33. Eberhart CG, Kaufman WE, Tihan T and Burger PC: Apoptosis, neuronal maturation and neurotrophin expression within medulloblastoma nodules. J Neuropathol Exp Neurol 60: 462-469, 2001.

34. Lavoie JF, Lesauteur L, Kohn J, Wong J, Furtoss O, Thiele CJ, Miller FD and Kaplan DR: TrKA induces apoptosis of neuroblastoma cells and does so via a p53-dependent mechanism. J Biol Chem 280: 29199-29207, 2005. 